Aim(s)/objectives To identify facilitating or prohibiting factors for HIV infected MSM when undertaking partner notification following HIV diagnosis.

Methods Semi structured interviews with ten newly diagnosed HIV MSM. All were recruited from a local NHS HIV outpatient service. Interviews were recorded verbatim and framework analysis was used to analyse the data.

Results Facilitating factors: There was a general acceptance and an awareness of necessity to initiate PN with immediacy, given the potential risk of onward transmission. Most participants expressed a "social responsibility "to inform partners of their HIV status if contactable, with a preference for disclosure through face to face contact if regular partner/s, but acknowledged that provider referral would be a useful option for non-regular or casual partners. Through "self-assessment of risk" most were able to identify the potential source of acquisition, and partners that could be "at risk" or infected. Prohibiting factors: Concerns about stigmatisation and criminalisation around disclosure of status remain key concerns, but participants particularly valued the support received from HCPs around addressing all aspects of PN.

Discussion/conclusion Important themes were identified that should be considered when supporting individuals in disclosing their HIV status to partners, providing a deeper understanding of the PN process from a patient's perspective and generating ideas that should be considered in future service provision and HIV PN studies.

\section{P65 SEXUAL BEHAVIOUR IN THE TIME PERIOD BETWEEN BEING TESTED FOR CHLAMYDIA AND RECEIVING TEST RESULT AND TREATMENT}

${ }^{1}$ Emma Harding-Esch* ${ }^{*}{ }^{1}$ Ellie Sherrard-Smith, ${ }^{1}$ Sebastian Suarez Fuller, ${ }^{1}$ Ana Harb ${ }^{1}$ Martina Furegato, ${ }^{2}$ Catherine Mercer, ${ }^{8} S$ Tariq Sadiq, ${ }^{4}$ Rebecca Howell-Jones, ${ }^{1}$ Anthony Nardone, ${ }^{5}$ Pam Gates, ${ }^{10}$ Amy Pearce, ${ }^{10}$ Frances Keane, ${ }^{6}$ Helen Colver, ${ }^{7}$ Achyuta Nori, ${ }^{8}$ Claire Dewsnap, ${ }^{8}$ Rebecca Schatzberger, ${ }^{9}$ Claudia Estcourt, ${ }^{9}$ Suba Dakshina, ${ }^{1}$ Catherine Dakshina, ${ }^{1}$ Catherine Lowndes. 'Public Health England, London, UK; ${ }^{2}$ University College London, London, UK; ${ }^{3}$ St George's, University of London, London, UK; ${ }^{4}$ Oxford School of Public Health, Oxford, UK; ${ }^{5}$ Royal Cornwall Hospital NHS Trust, Cornwall, UK; ${ }^{6}$ University Hospitals of Leicester NHS Trust, Leicester, UK; ${ }^{7}$ St George's Healthcare NHS Trust, London, UK; ${ }^{8}$ Sheffield Teaching Hospitals NHS Trust, London, UK; ${ }^{9}$ Barts Health NHS Trust, London, UK; ${ }^{10}$ Cornwall Sexual Health Service RCHT Clinic Team, Cornwall, UK

\subsection{6/sextrans-2015-052126.108}

Background/introduction There is a lack of data on the sexual behaviour of patients between being tested for chlamydia, receiving the test result, and being treated. This time-period may be important in the transmission of chlamydia, as infection could continue to be spread to sexual partners whilst awaiting the test result and treatment.

Aim(s)/objectives To investigate the sexual behaviours of patients between the time of being tested for chlamydia and receiving test result and treatment in order to investigate the benefits that a point-of-care test (POCT) might bring to clinical practice.

Methods We conducted a cross-sectional clinical audit of GUM clinic attendees. Clinic staff conducted a notes review of patients returning for chlamydia treatment following a positive chlamydia test result, and of age- and sex-matched chlamydia negatives. The data also served as an audit for the GUM clinics, following BASHH guidelines.

Results Five of nine GUM clinics approached participated, in July-December 2014. Data from 775 patients were included in analyses, 365 of whom were chlamydia-positive. Males with 2-4 partners, and those who reported never using a condom, were more likely to be chlamydia positive. For 21/143 (14.7\%) positive patients who provided data, last new sexual contact was in the period between test and treatment. Data were missing on condom use (22\%) and recent new partners (81\%).

Discussion/conclusion Patients continue to form new sexual partnerships whilst awaiting chlamydia test results, allowing for the possibility of infecting new sexual partners. POCTs which remove the test to treatment delay could prevent this onward transmission.

\section{Category: HIV prevention, PEPSE and PREP}

\section{P66 BASHH REGIONAL AUDIT OF PEPSE PROVISION IN THE NORTH-WEST OF ENGLAND}

${ }^{1}$ Jonathan Shaw*, ${ }^{1}$ Susanna Currie, ${ }^{2}$ Cara Saxon, ${ }^{1}$ Ashish Sukthankar. ${ }^{1}$ Manchester Centre for Sexual Health, Manchester, UK; ${ }^{2}$ University Hospitals of South Manchester, Manchester, UK

\subsection{6/sextrans-2015-052126.109}

Introduction Post-exposure prophylaxis following sexual exposure (PEPSE) to HIV is an established method of reducing HIV transmission.

Aims Review of the provision of PEPSE in North-West England against BASHH national auditable standards.

Methods Retrospective case note review of patients attending 15 genitourinary medicine clinics in the North-West England for PEPSE between 1st January 2013 and 31st December 2013. A maximum of 30 cases per centre were reviewed.

Results Of 203 cases reviewed 140 (67.0\%) were male, of whom 118 were MSM. Mean age was 31.5 years (range 15-75 years); 168 (82.8\%) were White British. HIV testing within 5 days of PEPSE initiation was recorded for 185 (91.1\%). Genitourinary departments starting PEPSE provided HIV testing for 103/112 (92.0\%) at baseline. Other departments starting PEPSE tested 10/91 (11.0\%). PEPSE was initiated for recommended indications in 187 cases (92.1\%) and 185 (91.1\%) were started within $72 \mathrm{~h}$ of exposure. Twenty-eight days of PEPSE was completed by 123 (60.6\%); 21 (10.3\%) discontinued early; $59(29.1 \%)$ did not have their treatment duration documented. STI screening was documented and accepted by 163 (80.3\%). A total of 98 (48.3\%) were HIV tested at 12 weeks post-PEPSE; all were negative. For those documented as completing PEPSE 76/123 (61.8\%) were HIV tested at 12 weeks post-PEPSE. At 6 months post-PEPSE 3 patients tested HIV-positive.

Conclusion PEPSE provision in the North-West met recommended standards for treatment initiation. However standards for PEPSE completion follow up and STI testing were not met. Documentation during follow up significantly impaired results and needs improvement.

\section{P67 PEP AWARENESS AMONGST A HIV-POSITIVE COHORT: WHO KNEW?}

Jonathan Shaw*, John Sweeney. Blackpool Sexual Health Services, Blackpool, UK

10.1136/sextrans-2015-052126.110 